\section{Alteración en la deambulación como forma de presentación atípica de infarto agudo de miocardio de localización inferior}

\section{Sr. Director:}

En los pacientes mayores de 85 años la cardiopatía isquémica cursa en un alto porcentaje de casos con síntomas distintos del dolor torácico. Entre ellos destacan la disnea, el sincope, el ictus y la confusión aguda (1-5). El primero en describir la presentación del infarto agudo de miocardio sin dolor torácico acompañante fue Herrick en 1912, desde entonces se han publicado muchos casos con presentaciones atípicas (1). Tras realizar una búsqueda en Medline (1966-2002), no hemos encontrado ningún caso que curse como empeoramiento clínico de una enfermedad neurológica previa en forma de incapacidad para la deambulación. Presentamos un caso con esta alteración en relación a infarto agudo de miocardio de localización inferior.

Se trata de un varón de 85 años de edad, con diabetes mellitus tipo 2 controlada con dieta y enfermedad de Parkinson leve (temblor de reposo, rigidez, bradicinesia y alteración de la marcha leve). No tenía antecedentes cardiológicos previos ni de demencia. Seguía tratamiento con levodopa, selegilina clorhidrato, piracetam y nimodipino. El paciente consulta porque su familiares notan desde hace 48 horas, un cuadro de mayor deterioro funcional para la deambulación en relación a su situación habitual. No refería sincopes, palpitaciones, disnea ni dolor torácico. No había realizado ningún cambio en su medicación habitual. En la exploración destacaba la ausencia de focalidad neurológica, se encontraba consciente y orientado, con presión venosa yugular normal, carótidas rítmicas y simétricas. La auscultación pulmonar presentó crepitantes bibasales. La auscultación cardiaca mostró tonos arrítmicos con extrasistolia frecuente sin soplos, abdomen sin hallazgos relevantes y extremidades con hallazgos compatibles con enfermedad de Parkinson: temblor de reposo, rigidez, bradicinesia y según su familia mayor alteración de la marcha de la que presentaba habitualmente.

El hemograma y la coagulación fueron normales. En la bioquímica encontramos glucosa $133 \mathrm{mg} / \mathrm{dl}$, creatinina $1,3 \mathrm{mg} / \mathrm{dl}$, CPK 240 UI/L con fracción MB de 97 UI/L, GOT 97UI/L, LDH $1.372 \mathrm{UI} / \mathrm{L}$, resto de parámetros dentro de la normalidad. La radiografía de tórax mostró cardiomegalia junto a redistribución vascular y signos compatibles con insuficiencia cardiaca izquierda. El electrocardiograma presentaba ritmo sinusal a 60 latidos por minuto, eje izquierdo, bloqueo auriculoventricular de primer grado, elevación del segmento ST en las derivaciones II, III y aVF con onda $\mathrm{T}$ negativa en las mismas junto a onda $\mathrm{Q}$ incipiente en III y aVF. El paciente fue ingresado con el diagnóstico de infarto agudo de miocardio de localización inferior de al menos 48 horas de evolución, por lo que no se realizó fibrinolisis. En las primeras doce horas del ingreso presentó edema agudo de pulmón con broncoespasmo, y en el electrocardiograma bloqueo auriculoventricular con conducción 2:1 y complejo QRS estrecho. Se le realizó ecocardiograma donde se apreció un ventrículo izquierdo levemente dilatado e hipertrofiado, con función sistólica global en el límite inferior de la normalidad, con cavidades derechas normales, aquinesia posterobasal e hipoquinesia inferior, válvula aórtica engrosada con buena apertura. Ausencia de derrame pericárdico y doppler de llenado mitral compatible con alteración de la relajación.

El paciente evolucionó favorablemente con tratamiento con broncodilatadores, diuréticos intravenosos y nitroglicerina. No presentó en ningún momento dolor torácico. Al alta presentaba onda $\mathrm{Q}$ en las derivaciones III y aVF con bloqueo auriculoventricular de primer grado con conducción 1:1 en el electrocardiograma. Según su familia, había recuperado su situación habitual en cuanto a la deambulación. Dadas sus características clínicas y funcionales se decidió de acuerdo con la familia no realizar otras pruebas complementarias ni tratamiento agresivo. Fue dado de alta con tratamiento conservador y revisiones periódicas en consulta.

El estudio Framinghan recoge que más del $25 \%$ de los pacientes con infarto agudo de miocardio cursan con síntomas distintos del dolor torácico, mientras que en otros estudios se habla de un tercio de los casos (2). Este porcentaje aumenta en edades más avanzadas. Los factores de riesgo más importantes para la aparición de manifestaciones atípicas son la diabetes mellitus, la edad avanzada, el sexo femenino, y los episodios previos de insuficiencia cardiaca ó ictus (3).

Entre las formas atípicas de presentación de cardiopatía isquémica, se encuentran los síntomas neurológicos, el síndrome confusional, la debilidad y la agitación (1-5). Las manifestaciones neurológicas más frecuentes son el sincope y el ictus, que suelen ser secundarias a bajo gasto cardiaco.

En nuestro caso, la única clínica que presentó el paciente se encontraba en relación con empeoramiento de su patología neu- 
rológica previa, con mayor dificultad para la deambulación, no refiriendo en ningún momento los síntomas habituales de la patología coronaria.

Los pacientes en los que la cardiopatía isquémica cursa sin dolor torácico, suelen consultar tardíamente con los servicios sanitarios. El diagnóstico no se realiza precozmente, esto conlleva un retraso para iniciar el tratamiento farmacológico, y en muchos casos no se efectúan terapias de reperfusión. Se ha objetivado, que esto duplica su riesgo de mortalidad en comparación con los enfermos en los que si aparece este síntoma (3).

Teniendo en cuenta la frecuente presentación atípica del síndrome coronario agudo en los pacientes ancianos, y la facilidad de acceso a pruebas no invasivas como el electrocardiograma, debería tenerse en cuenta esta patología cuando consulten por empeoramiento reciente de su situación clínica habitual para intentar realizar un diagnóstico precoz.

\section{D. Mañas, M. Montero ${ }^{1}$, E. Marchán, A. Benedicto ${ }^{1}$}

Servicios de Medicina Interna $y{ }^{I}$ Cardiología. Complejo Hospitalario de Ciudad Real

1. Thompson L, Wood C, Wallhagen M. Geriatric acute myocardial infarction: a challenge to recognition, prompt diagnosis, and appropiate care. Crit Care Nurs Clin North Am 1992; 4: 291-299.

2. Presentation of myocardial infarction in the elderly (editorial). Lancet 1986; 2: 1077-1078

3. Canto JG, Shlipak MG, Rogers WJ, Malmgren JA, Frederick PD, Lambrew CT, et al. Prevalence, clinical characteristics, and mortality among patients with myocardial infarction presenting without chest pain. JAMA 2000; 283: 3223-3229.

4. Gregoratos G. Clinical manifestations of acute myocardial infarction in older patients. Am J Geriatr Cardiol 2001; 10: 345-347.

5. Then KL, Rankin JA, Fofonoff DA. Atypical presentation of acute myocardial infarction in 3 age groups. Heart Lung 2001; 30: 285-293.

\section{Hematoma de los músculos rectos abdominales en relación con tratamiento de heparina de bajo peso molecular}

\section{Sr. Director:}

Diversos estudios han demostrado la eficacia de las heparinas de bajo peso molecular (HBPM) en la prevención y el tratamiento de la enfermedad tromboembólica venosa y del síndrome coronario agudo, tanto de la angina inestable como del infarto de miocardio sin onda $\mathrm{Q}(1-3)$.

Pese a los datos de seguridad, procedentes de los ensayos clínicos, se han descrito varios casos de hematomas espinales, retroperitoneales, musculares y cerebrales, relacionados con la administración de las heparinas de bajo peso molecular (4-7),

Recientemente hemos tenido ocasión de diagnosticar un caso de hematoma de los músculos rectos abdominales tras tratamiento con heparina de bajo peso molecular, cursó con anemización, hipotensión progresiva y la evolución fue finalmente fatal, pese a las medidas instauradas. Dada su rareza nos parece interesante presentarlo brevemente.

Mujer de 76 años de edad, con antecedentes de cardiopatía isquémica crónica e hipercolesterolemia. En las últimas semanas había presentado un cuadro de tos y dificultad respiratoria, siendo revisada en neumología, diagnosticándose de bronquitis aguda e instaurándose tratamiento con salbutamol, deflazacort, fluticaso- na y salmeterol.

En las últimas 24 horas presentó dolor torácico y aumento de la disnea. A la exploración física, la paciente estaba consciente, orientada y bien perfundida, Auscultación pulmonar: roncus y crepitantes de predominio basal. Auscultación cardiaca: Tonos rítmicos, normofrecuentes, sin soplos ni roces. Abdomen: blando y depresible, no doloroso, no se palpaban organomegalias. No edemas maleolares. T.A: $130 / 70 \mathrm{mmHg} 36,5^{\circ} \mathrm{C}$.

Laboratorio. Hemograma: 11.800 leucocitos con una distribución normal, $\mathrm{Ht}^{\circ}:$ 39,9\%, Hb: 13,4 gramos/dl, 239.000 plaquetas, actividad de protrombina del 100\%, fíbrinógeno: $364 \mathrm{mg} / \mathrm{dl}$. En la bioquímica, destacaba una CPK de 236 U/1, CPK MB de 23,4 $\mathrm{U} / \mathrm{I}$, troponina I: $2,81 \mathrm{ng} / \mathrm{ml}$ (normal hasta 0,06), dímero $\mathrm{D}$ de $5002 \mathrm{ng} / \mathrm{ml}$. ECG: se observó un descenso del espacio ST en toda la cara anterior, sin evidenciarse ondas Q.

Radiografía de tórax: Infiltrado alveolo- intersticial bilateral, de predominio en el hemitórax derecho. Fue diagnosticada de infarto de miocardio sin onda Q y de edema agudo de pulmón. Se inició tratamiento con nitroglicerina en perfusión, heparina sódica, furosemida y ácido acetil salicílico. A las seis horas se suspendió la heparina sódica, continuando con enoxaparina a dosis de $60 \mathrm{mg} / 12$ horas. Al suspenderse la heparina sódica el INR de la TTPA era de 1,57. En una analítica posterior la CPK ascendió hasta un valor máximo de $388 \mathrm{U} / 1$ y la troponina I de 7,03 ng/ml.

A las 24 horas de iniciarse el tratamiento con enoxaparina, presentó dolor abdominal en el hemiabdomen inferior, no se apreciaron datos de irritación peritoneal y el peristaltismo estaba conservado.

En el hemograma se objetivo un $\mathrm{Ht}^{\circ}$ de $26,5 \%$ y una hemoglobina de 9 gramos /dl, 167.000 plaquetas, actividad de protrombina del $100 \%$ y TTPA con un INR de 1,07 . Se solicitó una ecografía abdominal en el que se apreció una colección heterogénea de $8 \times 4 \times 2,5$ centímetros, en la pared abdominal anterior derecha, compatible con hematoma de pared (Fig. 1). Asimismo se observó otra colección de 1 x 1 x 2 centímetros en la pared abdominal anterior izquierda, compatible con hematoma de pared.

Se transfundieron 2 unidades de concentrados de hematíes. Una nueva radiografía de tórax mostró desaparición de los infiltrados pulmonares. La evolución posterior fue rápidamente desfavorable, presentando hipotensión y oligoanuria, sin responder a las medidas terapéuticas instauradas, falleciendo a las 48 horas de su ingreso. Cuando se ha presentado una complicación hemorrágica secundaria al tratamiento con heparinas de bajo peso molecular, la mayoría de los pacientes tenían factores de riesgo hemorrágico, como son una edad superior a 65 años, el uso de otros fármacos con riesgo hemorrágico (como los antiinflamatorios),

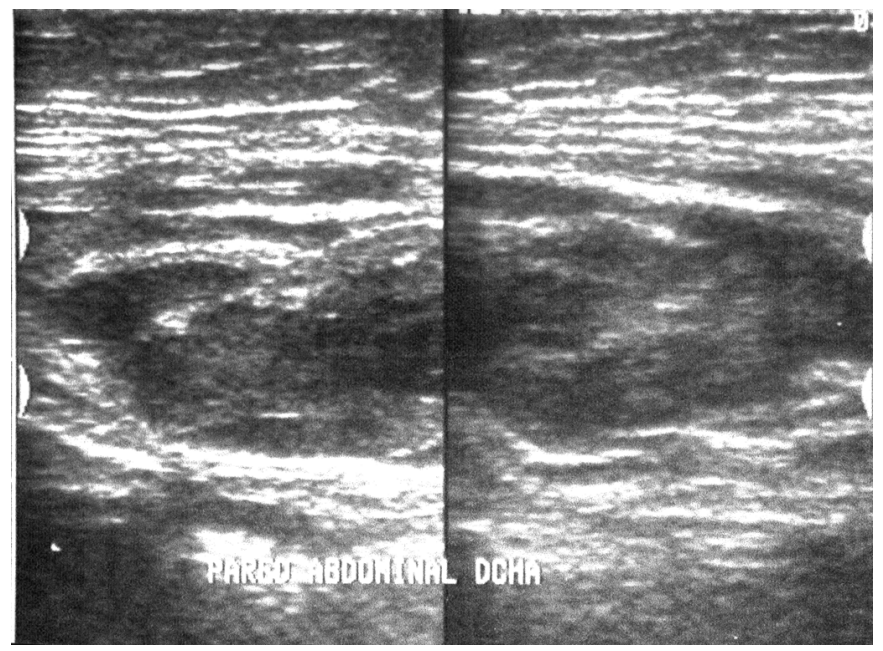

Fig. 1. 
una duración prolongada del tratamiento anticoagulante (superior a los 7 días), el empleo de dosis anticoagulantes, la realización de exploraciones diagnósticas y terapéuticas con riesgo hemorrágico, el antecedente de hemorragias previas y comorbilidad asociada con riesgo hemorrágico, que básicamente son la existencia de nefropatía, hepatopatía, hipertensión arterial, coagulopatía y úlcera gastrointestinal (8).

En nuestro caso, presentaba como factores de riesgo, la edad superior a 65 años y el empleo de heparina de bajo peso molecular a dosis anticoagulantes. En los adultos clínicamente estables no es necesario la monitorización de la actividad de la actividad anti-Xa de las heparinas de bajo peso molecular, sin embargo en los pacientes con factores de riesgo sería conveniente el ajuste de la dosis (9).

La seguridad terapéutica de las heparinas de bajo peso molecular aumentaría si se valorasen los factores de riesgo hemorrágico de los pacientes y se ajustas en las dosis en función del riesgo.

\section{F. Árbol Linde, F. Marcos Sánchez', M. Quintana Díaz, P. López Onega}

Servicios de Medicina Intensiva y de ${ }^{\prime}$ Medicina Interna. Hospital Nuestra Señora del Prado. Talavera de la Reina. Toledo

1. Leizorovicz A, Haugh MC, Chapuis FR, Samama MM, Boissel JP Low molecular weight heparin in prevention of perioperative thrombosis. BMJ 1992; 305: 913-920.

2. Lensing AW, Prins MH, Davidson BL, Hirsh J. Treatment of deep venous thrombosis with low- molecular- weight heparins. A meta-analysis. Arch Intern Med 1995; 155: 601-607.

3. Spinler SA, Nawarskas JJ. Low-molecular-weight heparins for acute coronary syndromes. Ann Pharmacother 1998; 32: 103-110.

4. Wysowsky DK, Talarico L, Bacsanyi J, Bostein P. Spinal and epidural hematoma and low-molecutar -weight heparin. N Engl J Med 1998; 338: 1774-1775.

5. Gómez de la Torre R, Milla Crespo A, Cadenas F, Fernández Bustamante J, Vázquez Castañón M. Hematoma retroperitoneal espontáneo inducido por enoxoparina a dosis terapéuticas. An Med Interna (Madrid) 2003; 20: $386-387$

6. Yusti Campo M, Lado Lado FL, Pérez Herbón M, Rodríguez Constenla I, Rodríguez López I. Hematoma de pared abdominal como complicación del uso de heparinas de bajo peso molecular. An Med Interna (Madrid) 2003; 20: 273-274.

7. Dickinson LD, Miller LD, Patel CP, Gupta SK. Enoxaparin increases the incidence of postoperative intracraneal hemorrhage when initiated preoperatively for deep venous thrombosis prophylaxis in patients with brain tumors. Neurosurgery 1998; 43: 1074-1081.

8. Vallano Ferraz A, Pedrós Cholvi C, Montané Esteva E, Bejarano Romero F, López Andrés A, Cereza García G et al. Hemorragias en pacientes tratados con heparinas de bajo peso molecular. Rev Clin Esp 2002; 202: 583-587.

9. Aguilar D, Golhaber S. Clinical uses of low-molecular-weight heparins. Chest 1999; 115: 1418-1423.

\section{Histiocitoma multicéntrico}

\section{Sr. Director:}

A continuación describimos el caso de una paciente diagnosticada de histiocitoma multicéntrico, con afectación ósea a nivel de articulación metacarpofalángica (MCF) y de partes blandas a nivel costal, asociación no descrita hasta el momento.

Paciente de 80 años, que desde hacía 6 meses presentaba astenia, anorexia y pérdida de $5 \mathrm{~kg}$ de peso, ingresó en nuestro servi- cio por fiebre de $38^{\circ} \mathrm{C}$ junto a dolor e inflamación del carpo y región tenar de la mano izquierda de 20 días de evolución. A la exploración TA 120/70 $\mathrm{mmHg}$, frecuencia respiratoria $18 \mathrm{rpm}$, frecuencia cardíaca $76 \mathrm{lpm}, \mathrm{t}^{\mathrm{a}} 38,5^{\circ} \mathrm{C}$. Inflamación y enrojecimiento de la articulación metacarpofalángica del primer dedo de la mano izquierda, y tres tumoraciones blandas, no adheridas de 1 a $1,5 \mathrm{~cm}$ de diámetro localizadas una en pared abdominal, otra debajo del apéndice xifoides, y otra en costado derecho. En la analítica destacaba leucocitosis de 32.000 cél $/ \mathrm{mm}$ (polimorfos $83 \%$, cayados $3 \%$ ), VSG de $41 \mathrm{mmHg}$ en la primera hora, PCR $6,67 \mathrm{mg} / \mathrm{dL}$, fosfatasa alcalina de 342 y GOT $173 \mathrm{UI} / \mathrm{mL}$. Resto del hemograma y bioquímica normales. Sedimento urinario con leve piuria y hematuria. Urocutlivos y hemocutlivos negativos. Serología a Brucella, VEB, CMV, toxoplasma, Mycoplasma, Chlamydia, fiebre Q negativos. Mantoux negativo. Radiografía de tórax normal. En la radiografía de manos se observaba osteolisis del borde externo del trapecio de la muñeca izquierda, junto a tumefacción de partes blandas del primer dedo y carpo de la misma mano. Se realizó artrocentesis y extracción del material purulento cuyo cultivo, para bacterias y mycobacterias, fueron negativos. En la ecografía abdominal se observaron adenopatías delante del tronco celíaco, detrás de la arteria hepática, en región interaórtico-cava, hilio hepático y retroperitoneo, confirmadas mediante TAC abdominal. En la gammagrafía ósea se advertía un aumento en la captación del trazador a nivel de la articulación MCF del primer dedo de la mano izquierda. Se administró tratamiento antibiótico empírico con cloxacilina 2 g/iv/8 horas y ceftriaxona $2 \mathrm{~g} / \mathrm{iv} / 24$ horas, y se realizó drenaje y biopsia de la zona tumefacta del dedo, junto a extirpación de las tumoraciones, costal y abdominal. En el examen macroscópico de las biopsias se encontró material necrosado, y al microsocopio una neoformación de estirpe mesenquimal con abundantes elementos linfocitarios y mitóticos, distribución celular anárquica, atípica, de aspecto maligno, con estroma laxo y escaso, con citoplasma amplio, eosinófilo, núcleo irregular y nucelolo prominente, compatible con histiocitosis maligna de alto grado. La artritis del dedo se resolvió y la enferma rechazó otro tratamiento, fue dada de alta y no acudió a consulta para seguimiento.

La histiocitosis fibrosa maligna es uno de los sarcomas de partes blandas que más frecuentemente afectan a los adultos (1); raramente se presenta en hueso y cuando lo hace suele localizarse en la metáfisis distal del fémur y proximal de la tibia, húmero y pelvis (2). Hasta ahora, sólo se han descrito en la literatura médica 4 casos de histiocitosis maligna que se encontraran en la articulación MCF como única localización (3-5). Lo novedoso de nuestro caso, se basa por una parte, en que la paciente presentaba una asociación de histiocitomas malignos no publicada hasta el momento, con afectación de hueso en articulación MCF, y partes blandas; y, por otra, en que el histiocitoma de la articulación MCF se complicó por una infección. El tratamiento del histiocitoma maligno óseo se realiza en función del grado de malignidad y de la extensión del tumor, los de bajo grado se tratan únicamente mediante resección quirúrgica amplia, y los de alto grado combinando resección y quimioterapia pre y postoperatoria, que incluye metotrexato a dosis altas $(2,6)$. El tratamiento del histiocitoma maligno de partes blandas se fundamenta en la resección quirúrgica amplia, aunque se puede administrar de forma conjunta radioterapia en caso de tumores de alto grado de malignidad o que tras la cirugía presenten restos neoplásicos en los bordes; aún se está investigando el papel de la quimioterapia en el tratamiento de los histiocitomas de partes blandas (7). La supervivencia del histiocitoma maligno óseo se encuentra cercana al $80 \%$ en tumores localizados de cadera (6); en cuanto al histiocitoma maligno de partes blandas, el porcentaje de supervivencia sin recidiva y el porcentaje total de supervivencia a los 5 y 10 años está en torno al 39 y $36 \%$, y al 50 y $43 \%$, respectivamente (7). 


\section{Hidalgo-Tenorio, M. P. Hernández, L. Hernández}

Servicio de Medicina Interna. Hospital Universitario Virgen de las Nieves. Granada

1. Asahi T, Kurimoto M, Kawaguchi M, Yamamoto N, Sato S, Endo S. Malignanat fibrous histiocytoma originating at the site of a previous fronto-temporal craniotomy. J Clin Neurosci. 2002; 9 (6): 704-8.

2. Delepine F, Delepine G, Belarbi L, Markowska B, Alkallaf S, Cornille H, Delepine N. Diagnosis and treatment of malignant bone fibrohistiocytoma Ann Med Interne (Paris) 2001; 152: 437-45.

3. Dock W, Hajek P, Wittich G, Kumpan W, Grabenwöger F. Primary malignant fibrous histiocytoma of a metacarpal bone: a new localization. Br. J. Rad. 1989; 62: 940-942.

4. Mutale CB, Patil PS, Patel JB. Malignant fibrous histiocytoma of the second metacarpal. J Hand Surg (BR) 1986; 11: 149-150.

5. Wulle C. Malignant soft tissue tumros of the hand: report of 2 malignant fibrous histiocytomas and 2 extraskeletal myxoid chondrosarcomas. Handchir Mikochir Plast Chir 1989; 21: 97-102.

6. Delepine F, Delepine G, Belarbi L, Markowska B, Alkallaf S, Cornille H, Delepine N. Diagnosis and treatment of malignant bone fibrohistiocytoma. Ann Med Interne (Paris) 2001; 152: 437-45.

7. Belal A, Kandil A, Allam A, Khafaga Y, El-Husseiny G, El-Enbaby A, et al. Malignant fibrous histiocytoma: a retrospective study of 109 cases. Am J Clin Oncol 2002; 25: 16-22.

\section{Urinoma y trombosis venosa profunda}

\section{Sr. Director:}

La enfermedad tromboembólica, y su manifestación más frecuente la trombosis venosa profunda (TVP), responden a múltiples causas, congénitas y adquiridas, que actúan con unos mecanismos fisiopatológicos comunes a todas ellas: lesión vascular, activación del proceso de coagulación y/o disturbios en la corriente sanguínea (estasis vascular) $(1,2)$. Por esta multiplicidad de causas y su alta incidencia encaja en el concepto de enfermedades prevalentes objeto de la atención y la competencia del médico internista (3).

Nos parece de interés presentar un enfermo con una causa inusual de TVP, un urinoma.

Varón de 73 años que presenta dolor en MID desde la raíz del muslo, de unos días de evolución que aumenta con la deambulación y tumefacción de todo el miembro. Como antecedentes personales encontramos: diabetes mellitus tipo 2. Fue intervenido de neoplasia vesical realizándose RTU con derivación urinaria tipo Briker el año anterior a su ingreso. Exfumador importante. Sin antecedentes de inmovilización o traumatismo previo.

En la exploración física se encontró una tumoración dolorosa, blanda de bordes difusos en FID. Tumefacción a nivel de MID con ligera cianosis del pie y ausencia de pulsos femoral, tibial posterior o pedio de forma homolateral.

Se realiza una Eco-Doppler que demostró la existencia de TVP en territorio de la vena iliaco-femoral derecha sin descartar la extensión a femoral superior o cava inferior. Se solicita ecografía abdominal y pélvica para valorar la tumoración. Se objetiva la presencia de colección hipoecogénica $6 \mathrm{~cm}-6 \mathrm{~cm}$ sin dependencia intestinal e hidronefrosis derecha de grado I, siendo sugerente de posible urinoma. Posteriormente se realiza TAC abdómino-pélvico visualizándose colección de liquido retroperitoneal a nivel de Ilio-psoas derecho con compresión del sistema venoso. Se decide punción de la masa bajo control radiológico, dejando drenaje evacuador. Tanto el aspecto, como las características bioquími- cas y citológicas del líquido eran compatibles con orina, no aislándose células neoplásicas. Tras el drenaje completo de la colección el paciente se encontró asintomático, desapareció el dolor y la tumefacción del miembro. Fue dado de alta con tratamiento anticoagulante durante 3 meses, sin nuevo episodio posterior de TVP.

Un Urinoma es una extravasación encapsulada de orina, secundaria a pequeñas pérdidas por traumatismos (sobre todo quirúrgicos) o que puede ocasionarse espontaneamente, favorecido por la presencia de una obstrucción, siempre que se mantenga la función renal (4-6).

La extravasación de orina produce lipolisis y estimula una intensa reacción fibrosa, que provoca una encapsulación (4). Suele permanecer asintomático, producir molestias vagales o franco dolor si se complica con infección. El diagnóstico se confirma con pruebas de imagen -ecografía y TAC (7)- y se resuelve en muchas ocasiones con drenaje percutáneo.

En una búsqueda bibliográfica en Medline (base de datos med-line), no hemos encontrado descritas la TVP como complicación de la presencia de urinoma.

En nuestro enfermo, el mecanismo fisiopatológico evidente era la dificultad del retorno venoso, habiéndose descartado por las pruebas de imagen y evolución clínica posterior, una recidiva tumoral.

\section{Pretel Serrano, I. C. Sánchez Fuentes, A. Alemán Lorenzo, F. Núñez Martínez}

Servicio de Medicina Interna. Hospital General Universitario Morales Meseguer. Murcia

1. Rodríguez A, Rodríguez R, Estelles F. Trombosis venosa profunda y cáncer: Actitud diagnóstica. An. Med Interna (Madrid) 2002; 19: 657658 .

2. Lensing AW, Prandoni P. Deep-vein thrombosis. Lancet 1999; 353: 479485.

3. Conthe P, Vilardel M. Medicina Interna y enfermedades prevalentes. E caso de la insuficiencia cardiaca. Rev Clin Esp 2002; 202 63-65.

4. Rohner S, Tuchschmd Y, Graber P. Urinoma Périrénal. Ann Urol (Paris) 1994; 28: 259-264.

5. Ben Slama Mr, Salomon L, Hoznek A, et al. Extraperitoneal laparoscopic repair of ureteropelvic junction obstruccion: initial experience in 15 cases. Urology 2000; 56: 45-48.

6. Regalado Pareja R, Huguet Pérez J, Errando Smet C, Lima Bx, Chechile Toniolo G, Villavecencio Maurich H. Sustitución vesical ortotópica: III Resultados funcionales y complicaciones en los pacientes con Neovejiga ileal tipo Studer. Arch Esp Urol 1997; 50: 234-241.

7. Gayer G, Zissin R, Apter S, et al. Urinomas caused by ureteral injuries: CT appearance. Abdom Imaging 2002; 27: 88-92.

Necesidad de seguimiento endoscópico en la gastritis crónica atrófica con hiperplasia de células neuroendocrinas

\section{Sr. Director:}

La hiperplasia gástrica de células neuroendocrinas en la gastritis crónica atrófica está asociada al posible desarrollo de un tumor carcinoide gástrico (1).

Presentamos el caso de una mujer de 27 años de edad con hipotiroidismo autoinmune, gastritis crónica atrófica y anemia 
perniciosa. El estudio histológico realizado en el momento del diagnóstico mostró la presencia de hiperplasia de células neuroendocrinas en el fundus gástrico. Ante este hallazgo, se planteó la necesidad de establecer un programa de seguimiento endoscópico para la detección precoz del tumor carcinoide gástrico, si bien, no se conoce el intervalo de tiempo óptimo y seguro.

La gastritis crónica atrófica tipo A está asociada al 75 por ciento de los tumores carcinoides gástricos, los cuales se originan en las células enterocromafines del fundus. La prevalencia del tumor carcinoide en la anemia perniciosa varía entre el 1-2 por ciento (3). Estos pacientes presentan hipoclohidria e hipergastrinemia, lo que representa un estímulo para la proliferación de células neuroendocrinas (3). Sin embargo, parece que es la duración de la hipergastrinemia, más que la propia concentración sérica, la que se correlaciona mejor con el riesgo de desarrollo del carcinoide. La hiperplasia de células neuroendocrinas (Fig. 1) está presente de forma casi constante en los tumores carcinoides asociados a gastritis crónica atrófica, considerándose así una lesión premaligna. La incidencia de estos tumores, en presencia de hiperplasia, en el seguimiento endoscópico de los pacientes con anemia perniciosa, oscila entre 1-7\% (2). Esta lesión histológica también se puede observar en los carcinoides asociados al síndrome Zollinger-Ellison, en los pacientes con Neoplasia Endocrina Múltiple tipo I (MEN I).

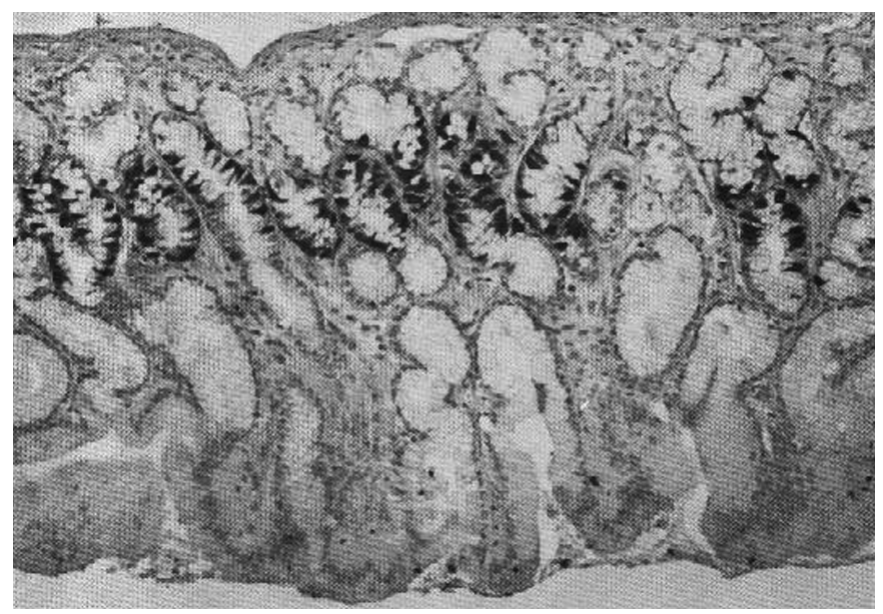

Fig. 1. Técnica de Cromogranina. Hiperplasia extensa de células G.

Otros factores, además de la hipergastrinemia, podrían jugar un papel determinante del grado de hiperplasia. Se han descrito casos de regresión del tumor tras antrectomía y reducción de los niveles de gastrina (2), si bien, también se han comunicado casos espontáneos.

En conclusión, se impone la necesidad de establecer un protocolo de seguimiento endoscópico de estas lesiones histológicas premalignas para la detección precoz del desarrollo del tumor carcinoide. El screening endoscópico conlleva un aumento en el diagnóstico de tumores pequeños con buen pronóstico incluso tras resección endoscópica local (4). Diferentes estudios prospectivos $(5,6)$ han propuesto intervalos de tiempo de 4-5 años en el seguimiento de los pacientes con hiperplasia o displasia leve o moderada. No obstante, se necesitan nuevos estudios a largo plazo con mayor número de pacientes para definir el intervalo óptimo de tiempo para el diagnóstico temprano del tumor carcinoide gástrico.

\section{R. Segoviano Mateo, R. Cigüenza Gabriel, R. Torres Gárate, B. Valle Borrego, J. Antolín Arias}

Servicio de Medicina Interna I. Hospital Clínico San Carlos. Madrid
1. Klaus J Lewin, Henry D Appelman. Endocrine cell proliferations of the stomach. Atlas of Tumor Pathology. Armed Forces Institute of Pathology (AFIP) 1995; 18: 331-356.

2. Sjöblom SM, Sipponen P, Järvinen H. Gastroscopic follow-up of pernicious anemia patients. Gut 1993; 34: 28-32.

3. Gilligan CJ, Lawton GP, Tang LH et al. Gastric carcinoid tumors: the biology and therapy of an enigmatic and controversial lesion. Am J Gastroenterol 1995; 90: 338-52.

4. Sculco D, Bilgrami S. Pernicious anemia and gastric carcinoid tumor: case report and review. Am J Gastroenterol 1997; 92 (8): 1378-80.

5. Armbrecht V, Stockbrügger RW, Rode J, et al. Development of gastric dysplasia in pernicious anemia: a clinical and endoscopic follow-up study of 80 patients. Gut 1990; 31: 1105-1109.

6. Edith Lahner, Pietro Caruana, Giancarlo D'Ambra, et al. First endoscopic-histologic follow-up in patients with body-predominant atrophic gastritis: when should it be done? Gastrointestinal endoscopy 2001; 53 : 443-8.

\section{Artritis tuberculosa periférica}

\section{Sr. Director:}

Mujer de 86 años, con único antecedente personal de interés, la intervención de prótesis en cadera derecha por fractura postraumática de cuello femoral dos años antes.

Acude a consulta por dolor de 3 meses de evolución de características inflamatorias en hombro derecho con impotencia funcional, acompañado de astenia y sudoración nocturna. En la exploración se encuentra dolor y limitación a la movilidad en hombro derecho con tumefacción. La analítica general presenta elevación de reactantes de fase aguda con una velocidad de sedimentación general (VSG) de $60 \mathrm{~mm}$ en la primera hora y una proteína $\mathrm{C}$ reactiva (PCR) de $21 \mathrm{mg} / \mathrm{dl}$, con hemograma y bioquímica normal. En la resonancia nuclear magnética (RNM) de dicho hombro se aprecia derrame de líquido articular con señal muy heterogénea e imágenes nodulares puntiformes en su interior sugestivas de sinovitis inflamatoria (Fig. 1). Se realiza artrocentesis obteniendo líquido sinovial con 21.000 células $/ \mathrm{mm}^{3}$ ( $80 \%$ polimorfonucleares), 4,9

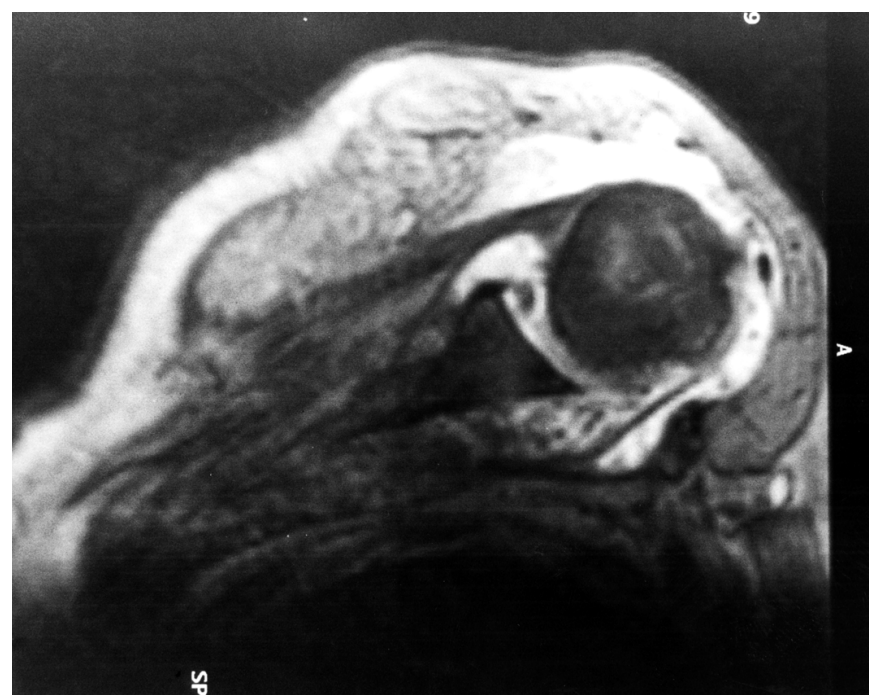

Fig. 1. RNM de hombro derecho axial potenciado en T2*: importante derrame articular con paso de líquido a la bolsa subacromiodeltoidea y presencia de imágenes puntiformes hipointensas en todas las secuencias compatible con bursitis en grano de arroz. 
gr/dl de proteínas, glucosa $56 \mathrm{mg} / \mathrm{dl}$, ADA 40 U/L sin hallazgo de cristales y con cultivo de Gram, flora bacteriana y hongos negativo. En cultivo de Löwenstein-Jensen se obtiene crecimiento para Mycobacterium tuberculosis. Se inició tratamiento tuberculostático con evolución excelente, sin objetivarse complicaciones.

La incidencia global de tuberculosis ha disminuído debido al desarrollo de las condiciones de vida, nutricionales y sanitarias de la población, con un descenso de la tuberculosis pulmonar, pero la localización osteoarticular sigue permaneciendo invariable con una edad de presentación por encima de los 40 años. La artritis tuberculosa periférica (ATP) tiene una incidencia de alrededor de 2,4\% (1); representa junto con las formas axiales y osteoarticulares en general, la tercera forma extrapulmonar en frecuencia, tras la renal y linfática. Las articulaciones de carga son mas comúnmente afectadas con predominio en rodillas y caderas. Como factores de riesgo deben considerarse factores generales, como enfermedades sistémicas autoinmunes, junto con la toma de corticoides e inmunosupresores y factores locales como infiltraciones intraarticulares y traumatismo. La dificultad en el diagnóstico al inicio de la enfermedad es debido a su sintomatología poco específica, pudiendo llegar a retrasarse hasta más de un año, sobre todo en aquellos casos sin antecedentes de tuberculosis pulmonar, tanto previa como actual (2). La sintomatología puede presentarse como un dolor crónico no intenso, tumefacción, atrofia muscular y tendencia a la anquilosis, acompañado de clínica general como febrícula, astenia, anorexia, pérdida de peso y sudoración nocturna (3).

El diagnóstico diferencial se llevará a cabo con aquellos procesos que producen monoartritis crónica como artritis reumatoide, artritis infecciosas bacterianas y micótica, artropatía neuropática, distrofia simpático refleja, osteonecrosis aséptica y sinovitis vellonodular pigmentaria principalmente. Los estudios analíticos elementales muestran pocas o ninguna alteración. Puede haber una discreta anemia microcítica e hipocrómica, con una ligera leucocitosis y una VSG discretamente elevada. La intradermorreacción de Mantoux suele ser francamente positiva. Las características del líquido sinovial son de una celularidad inflamatoria (entre 10.000 y 20.000 células) con predominio de polimorfonucleares, rico en proteínas y bajo en glucosa, siendo en ocasiones indetectable (4). Los hallazgos radiológicos aparecen tardíamente y son inespecíficos para el diagnóstico, independientemente del estadio en que se encuentren. La osteoporosis yuxtaarticular y el engrosamiento de partes blandas son los datos iniciales más frecuentes, para presentar posteriormente pinzamiento de la interlínea articular con quistes subcondrales que pueden dar lugar a pequeñas erosiones en los bordes articulares (5). La tomografía axial computarizada y la RNM muestran lesiones sugestivas de lesión articular con mayor precocidad que la radiología convencional y permite realizar el diagnóstico diferencial con otros procesos infecciosos (6).

El diagnóstico de artritis tuberculosa se realizará con la demostración del gérmen en el líquido sinovial o estructuras articulares mediante los diferentes métodos de tinción o el cultivo del mismo (7). Se procederá a la búsqueda en muestras de otras localizaciones como esputo, orina y jugo gástrico. La biopsia sinovial detectará la presencia de granulomas con necrosis caseosa en el tejido sinovial aunque el hallazgo de una biopsia negativa no excluye el diagnóstico.

El tratamiento se basa fundamentalmente en medicación antituberculosa y medidas ortopédicas con buenos resultados dependiendo de la instauración precoz de los mismos.

\section{M. Arias Miranda, F. J. Nuño Mateo, J. Babío Herráiz', J. Noval Menéndez, D. Galiana Martín}

Servicio de Medicina Interna y ${ }^{1}$ Reumatología. Hospital de Cabueñes. Gijón
1. Sánchez J, Aspe B, Graña J, Atanes A, Galdo F. Artritis tuberculosa periférica en Galicia. Med Clin (Barc) 1990: 88-91.

2. Mera AJ, Mosquera A, Iglesias G, Montoya A, Galdo F, García E. Tuberculosis articular periférica: estudio de 20 casos con biopsia sinovial. Med Clin ( Barc) 1985; 85: 605-608.

3. Hugh G, Lifeso RM. Tuberculosis of Bone and Joints. J Bone and Joint Surg, 1996; 78A: 288-298.

4. García JJ, Muñoz ML. Enfermedad de Lyme. Infecciones osteoarticulares por micobacterias, Brucella, hongos y parásitos. En: Alonso A, Alvaro-Gracia JM, Andreu JL, el at. (eds). Manual S.E.R. de las enfermedades reumáticas $3^{\mathrm{a}}$ edición. Madrid, 2000: 523-530.

5. Uriel S, Muguerza I, Echevarría F, López R, Amor T. Tuberculosis osteoarticular. Presentación de 54 casos. Rev Clin Esp 1979; 155: 277-281.

6. Hong SH, Kim SM, Ahn HW, Chung Hw, Shin MJ, Kang HS. Tuberculosis versus pyogenic arthritis: MR imaging evaluation. Radiology 2001; 218: 848-853.

7. García A. Tuberculosis de articulaciones periféricas. En: Pascual E, Rodríguez V, Carbonell J, Gómez-Reino JJ. Tratado de Reumatología. Madrid: Arán, 1998: 1329-1339.

\section{Empiema crónico por Pseudomonas aeruginosa}

\section{Sr. Director:}

El género Pseudomonas está constituido por bacilos gramnegativos muy difundidos en la naturaleza. Aunque pueden colonizar al ser humano y se describen bacteriemias, neumonías, endocarditis, osteomielitis, meningitis, otitis externas, enterocolitis necrotizante, infecciones urinarias y de piel, rara vez ocasionan enfermedad en el individuo sano (1). La afección pleural es algo excepcional. Se presenta un caso de empiema por Pseudomonas aeruginosa, con el interés añadido de lo inusual en la forma de presentación.

Varón de 76 años con antecedentes de EPOC severo, cor pulmonale, diabetes mellitus e insuficiencia renal crónica. Presenta cuadro de dos semanas de disnea progresiva con tos seca y edemas. A la exploración física hipoventilación en base izquierda y edemas bilaterales hasta rodillas. En los últimos 6 meses ingresó en tres ocasiones en distintos centros en los que se observó en la radiografía de tórax un derrame pleural izquierdo; aislándose Pseudomonas aeruginosa en esputo un mes antes. Recibió diversos ciclos de antibióticos y esteroides. En estudios complementarios destacar $\mathrm{Hb} 8,9 \mathrm{~g} / \mathrm{dl}, 7.800$ leucocitos con $77 \%$ de segmentados, creatinina $2,4 \mathrm{mg} / \mathrm{dl}$, urea $128 \mathrm{mg} / \mathrm{dl}$, LDH $620 \mathrm{UI} / 1$ (313-618), VSG $90 \mathrm{~mm} / \mathrm{h}, \mathrm{pO}_{2} 43$ y pCO 2 51. En TAC torácico se observa un derrame pleural izquierdo loculado y atelectasia parcial del lóbulo inferior izquierdo. Se realizó toracocentesis obteniendo líquido purulento con 70.200 leucocitos y glucosa $5 \mathrm{mg} / \mathrm{dl}$. En líquido pleural y esputo se aisló Pseudomonas aeruginosa sensible a ciprofloxacino, ceftazidima, gentamicina, aztreonam e imipenen. Con el diagnóstico de empiema crónico por Pseudomonas aeruginosa se instauró tratamiento antibiótico con ceftazidima más ciprofloxacino y drenaje a través de tubo de tórax con mala evolución falleciendo al mes.

Los patógenos que se aíslan con mayor frecuencia en los empiemas son estafilococos y estreptococos junto con bacterias anaerobias (2). La neumonía por Pseudomonas aeruginosa es una infección infrecuente y la afectación pleural excepcional. Salavert y cols. (3) recogen 17 episodios de bacteriemias por Pseudomonas aeruginosa en pacientes con infección por VIH con 7 casos de neumonía y ninguno de empiema. Graczyk y cols. (4) recogen 66 neumonías por Pseudomonas aeruginosa con 7 casos de empiemas como predictores de fracaso terapéutico.

La presencia de un empiema crónico loculado prácticamente asintomático, como el que se presenta, supone una extrañeza por 
sí mismo y más si tenemos en cuenta el agente causal. A este respecto Sahn y cols. (5) describen como tres pacientes que recibían corticoides desarrollaron un empiema, todos estaban afebriles y con mínima sintomatología.

El mecanismo patogénico que produce la infección pleural por Pseudomonas es por diseminación hematógena en el transcurso de una sepsis (SIDA, trasplantados, neutropenias severas...) o por aspiración no bacteriémica en pacientes con EPOC, insuficiencia cardiaca o que hayan recibido antibióticos de amplio espectro o esteroides de forma crónica (1), circunstancias que se dan en nuestro paciente, en el que pensamos que a partir de un foco primario pulmonar se desarrolló, enmascarada por los corticoides, la afectación pleural.

El tratamiento debe incluir drenaje pleural y un antibiótico betalactámico con actividad antipseudomonas más un aminoglucósido $(6,7)$.

En conclusión, la ausencia de fiebre o síntomas torácicos no debe excluir la consideración diagnóstica de un empiema y especialmente si el paciente ha recibido tratamiento con corticoides.

\section{A. Morán Blanco, J. Guerra Laso, M.A. Remacha Esteras ${ }^{1}$}

\section{Medicina Interna. ${ }^{1}$ Microbiología. Hospital de León}

1. Mallolas Masferrer J, Vila Estapé J. Infecciones causadas por Pseudomonas y otros bacilos gramnegativos no fermentadores. Medicine 2002; 64: 3398-3407.

2. Mordasini C, Krneta A, Baumann HR. Diagnosis and therapy of pleural empyema Schweiz Med Wochenschr 1988; 118: 1633-40.

3. Salavert Lletí M, Navarro Ibáñez V, Roig Rico P, Bretón Martínez JR, Ponz Díez F, López Aldeguer J, Gobernado Serrano M. Bacteriemia por Pseudomonas aeruginosa en pacientes con infección por el VIH: estudio clinicoepidemiológico de 17 episodios. Rev Clín Esp 1996; 196: 684-691.

4. Graczyk J, Szmidt M, Fijalkowski M, Minc P, Cieslak M. Pseudomonas aeruginosa pneumonia in patients treated at the Hospital for Chest Diseases. Pneumonol Alergol Pol 2000; 68 (3-4): 101-8.

5. Sahn SA, Lakshminarayan S, Char DC «Silent» empyema in patients receiving corticosteroids. Am Rev Respir Dis 1973; 107: 873-876.

6. Rahal JJ Jr. Rationale for use of antimicrobial combinations in treatment of gram-negative infections. A review of recent reviews. Am J Med 1983; 75 (2A): 68-71.

7. Light RW. Porcel JM. Derrame pleural paraneumónico y empiema. Rev Clin Esp 2000; 115: 384-391.

\section{Leucemia linfoide aguda tras cáncer de mama y fiebre Q}

\section{Sr. Director:}

La leucemia linfoide aguda (LLA) en adultos que han sufrido una neoplasia previa es poco frecuente, y los factores de riesgo que contribuyen a la aparición de la LLA en estos pacientes no son bien conocidos (1). Se ha propuesto que el tratamiento mutagénico previo o una predisposición biológica al cáncer podrían desempeñar un papel en la aparición de la LLA en pacientes que han sufrido una neoplasia previa (1). Por otra parte, existen evidencias epidemiológicas que sugieren que las infecciones pueden estar implicadas en la etiología de algunos tipos de LLA $(2,3)$. Presentamos el caso de una paciente previamente tratada por un cáncer de mama que sufrió una LLA tras una infección aguda por Coxiella burnetii.

Una mujer de 53 años acudió por presentar, desde 15 días antes, fiebre, cefalea y astenia. Cinco años antes había sido trata- da con éxito por un cáncer de mama con cirugía, radioterapia y 6 ciclos de quimioterapia de tipo CMF (ciclofosfamida, metotrexate y 5-fluorouracilo). Dos meses antes, en una revisión, estaba libre de enfermedad y su hemograma y bioquímica sérica no mostraban alteraciones.

En la consulta actual su hemograma mostraba valores normales. La bioquímica sérica mostraba elevados los valores de LDH (620 U/L) y proteína C-reactiva (PCR: $29 \mathrm{mg} / \mathrm{dL}$ ). Los estudios encaminados a la detección de agentes infecciosos, incluyendo la serología de fiebre Q, dieron resultados negativos. Dos semanas después los síntomas persistían y la paciente fue ingresada (día 0). Su hemograma mostraba 7.230 leucocitos $/ \mathrm{mL}$ con un $40 \%$ de linfocitos $(2.920$ linfocitos/mL), 178.000 plaquetas/mL y una hemoglobina de 13,4 g/dL. La LDH era de 996 U/L y la PCR de 86 mg/dL. El día +1 todos los síntomas, excepto la fiebre, habían desaparecido. El día +8 la paciente seguía con fiebre y el estudio serológico puso de manifiesto un título de IgG frente a Coxiella burnetii (fase II) de 640, demostrando la existencia de una seroconversión indicativa de infección reciente por dicho agente. El hemograma del día +10 mostró 9.770 leucocitos $/ \mathrm{mL}$ con 5.300 linfocitos $/ \mathrm{mL}$. El día +15 la paciente aún tenía fiebre y el estudio de la médula ósea reveló la presencia de un $69 \%$ de blastos con morfología L2. Estos blastos tenían un cariotipo normal y el siguiente inmunofenotipo: CD45+, CD34+, CD19+, CD10-, CD3-, MPO-, HLA-DR+, CD79a+, CD15+, correspondiente a una LLA-proB. El estudio de sangre periférica reveló la presencia de un $40 \%$ de blastos. El día +22 el hemograma mostraba 15.730 leucocitos $/ \mathrm{mL}, 125.000$ plaquetas $/ \mathrm{mL}$ y una hemoglobina de 11,6 $\mathrm{g} / \mathrm{dL}$. La LDH era de $1.283 \mathrm{U} / \mathrm{L}$. Se administró doxiciclina (desde el día +16) y terapia citotóxica (desde el día +22). La fiebre desapareció el día +23 . Ocho semanas más tarde la paciente falleció debido a una sepsis durante el tratamiento de consolidación.

Nuestra paciente sufrió un síndrome febril asociado a una infección aguda por Coxiella burnetii seguido de una LLA. La asociación entre la LLA y la fiebre Q no ha sido comunicada previamente. En el caso presente la rápida sucesión de fiebre Q y LLA podría ser casual. No obstante, hay hipótesis que implican a las infecciones en la etiología de la LLA (2,3). Estas hipótesis proponen un modelo de dos pasos: $1^{\circ}$ ) una mutación o daño cromosómico generaría un clon celular preleucémico y $2^{\circ}$ ) la respuesta a una infección provocaría la expansión de dicho clon y la aparición de la LLA $(2,4)$. Se ha propuesto que las exposiciones a agentes infecciosos son estímulos proliferativos para los linfocitos $\mathrm{B}$, pudiendo conferir ventaja proliferativa a células mutadas $(3,4)$ o promover las alteraciones genéticas adicionales necesarias para la aparición de la leucemia $(2,4)$. En nuestro caso, la respuesta a la infección por Coxiella burnetii podría haber sido el desencadenante de la LLA en una paciente que era posiblemente susceptible a la leucemia debido al tratamiento genotóxico previo y/o a una predisposición biológica a la neoplasia (1). Creemos que, para contribuir a aclarar el hipotético papel de los agentes infecciosos en la etiología de la leucemia aguda, podría ser interesante investigar la existencia de infecciones recientes en pacientes con LLA.

J. Bartolomé Álvarez, J. R. Romero Macías', S. Lorente Ortuño, A. Marín Ors, E. Heredero Gálvez

Laboratorio de Microbiología y ${ }^{1}$ Servicio de Hematología. Hospital General Universitario. Albacete

1. Pagano L, Pulsoni A, Mele L, Leone G. Clinical and epidemiological features of acute lymphoblastic leukemia following a previous malignancy. Leuk Lymphoma 2000; 39: 465-75.

2. Greaves M. Childhood leukaemia. BMJ 2002; 321: 283-7.

3. Vineis P, Miligi L, Crosignani P, Davico L, Fontana A, Masala G, et al. Delayed infection, late tonsillectomy or adenoidectomy and adult leukaemia: a case-control study. Br J Cancer 2003; 88: 47-9.

4. Greaves MF. Aetiology of acute leukaemia. Lancet 1997; 349: 344-9. 


\section{Intoxicación familiar accidental por ingesta de Datura stramonium}

\section{Sr. Director:}

La Datura stramonium, también conocida como "higuera del infierno", "flor de la trompeta"e "higuera loca", pertenece a la familia de las Solanaceae, y crece en zonas mediterráneas. Contiene alcaloides con intenso efecto anticolinérgico, como son la atropina, escopolamina e hiosciamina, empleándose como planta medicinal así como droga alucinógena $(1,2)$. La intoxicación con ella puede producir un cuadro anticolinérgico potencialmente mortal, aunque en general el pronóstico es bueno (3-5). Presentamos los casos de una intoxicación accidental de una familia por la ingesta de semillas de Datura stramonium.

Cinco miembros de una familia acuden al Servicio de Urgencias por presentar sintomatología consistente en sequedad de boca marcada, dificultad para iniciar la micción, sensación de mareo y visión borrosa, sumándose en dos de ellos alteración de la marcha. Los síntomas comienzan una hora después de ingerir unas "gachas" preparadas con harina de maíz, que según refería el padre, ésta estaba mezclada con unas semillas de una planta conocida como "higuerica". En la exploración física todos los pacientes presentaban midriasis reactiva bilateral y sequedad de mucosas, sumándose ataxia de la marcha en dos de ellos. La familia ingresa con clínica de síndrome anticolinérgico tras ingesta de harina de maíz contaminada por unas semillas, que ante las explicaciones del padre y el cuadro clínico que presentaron, se sospechó que pudiera corresponder a la ingesta de Datura stramonium, siendo identificada la misma por el paciente al visualizar unas fotografías de dicha planta. Los pacientes en las primeras 12-24 horas del ingreso quedaron asintomáticos, sin aparición de ninguna complicación, no precisando de fármacos anticolinesterásicos procediéndose al alta a las 48 horas del ingreso.

La intoxicación por Datura stramoniun es más común en aquellas personas que consumen esta planta como droga alucinógena $(6,7)$, siendo la intoxicación accidental más infrecuente, dándose ésta sobre todo en edad pedriátrica. El diagnóstico normalmente es clínico y debe iniciarse un tratamiento precoz consistente en descontaminación intestinal, tratamiento de soporte y anticolinesterásicos si precisa por la situación clínica (8). El diagnóstico diferencial con otras intoxicaciones que producen síndrome anticolinérgico es amplio, incluyendo fármacos (atropina, antidepresivos tricíclicos, neurolépticos, antihistamínicos, amantadina...), setas, otras plantas (Atropa Belladona, Hyosciamus Níger). En nuestro caso se llegó al diagnóstico tras una detallada historia clínica y tras la identificación de uno de los miembros de la familia de la planta al mostrarle una imagen de la misma obtenida por internet, pudiendo ser este medio en el caso de intoxicaciones por plantas y setas de vital importancia, dada la diferente toxicidad en cada caso.

\section{J. M. Calbo Mayo, M. A. Barba Romero, L. Broseta Viana, F. Medrano González}

Servicio de Medicina Interna. Hospital General Universitario. Albacete

1. Cartanon López L, Martínez Badás JP, Lapena López de Armentia S, Gómez Mora J, García Arias ML. Intoxicación por Datura stramonium. An Esp Pediatr 2000; 53 (1): 53-5.

2. Roblot F, Montaz L, Delcoustal M et al. Datura stramonium poisoning: the diagnosis is clinical, treatment is symptomatic. Rev Med Intern (Paris) 1995; 16: 187-190.

3. Thabet H, Brahmi N, Amamou M, Ben Salah N, Hedhili A, Yacoud M. Datura stramonium poisonings in humans. Vet Hum Toxicol 1999; 41 (5): 320-1.
4. Jiménez-Mejías ME, Fernández A, Montaño-Díaz M, González de la Puente MA. Síndrome anticolinérgico por envenenamiento por Datura stramonium. Med Clin (Barc) 1991; 97: 237.

5. Alcaraz García SF, Girón Úbeda JM, Delgado López F, Gómez García AJ. Midriasis por contacto accidental con estramonio (Datura stramonium). Med Clin (Barc) 1999; 113: 156.

6. Baselga JM, Pigron C, Martínez-Vázquez JM. Datura stramonium: ¿un antiguo alucinógeno en auge? Med Clin (Barc) 1985: 84: 715.

7. Tiongson J, Salen P. Mass ingestion of Jimson Weed by eleven teenagers. Del Med J 1998; 70: 971-6.

8. Rodgers GC, Von Kanel RL. Conservative treatment of Jimson weed ingestion. Vet Hum Toxicol 1993; 35: 32-33.

\section{Embolia pulmonar tras fleboesclerosis de varices en extremidad inferior}

\section{Sr Director:}

La fleboesclerosis o esclerosis farmacológica de varices secundarias y varículas en las extremidades inferiores es un procedimiento terapéutico frecuente, de práctica a nivel de consulta externa y en general de bajo riesgo. Presentamos el caso de una paciente que tras una fleboesclerosis de varices desarrolló un cuadro documentado del tromboembolismo pulmonar (TEP).

Paciente de 58 años, sexo femenino y afecta de varices secundarias, varículas y telangiectasias en extremidades inferiores oligosintomáticas. Sin antecedentes personales de interés, colecistectomía hacía 5 años con analítica y estudio preoperatorio normal. Constitución y peso normal, no fumadora y sin factores de riesgo de enfermedad tromboembólica venosa.

El 23 de abril del 2002 se realizó en consulta de Cirugía Cardiovascular fleboesclerosis de varices en extremidad inferior izquierda; inyectándose $3 \mathrm{ml}$ de polidocanol al 0,5\% y realizándose después vendaje semicompresivo. El procedimiento fue sin incidentes y la paciente abandonó la consulta en buen estado.

A las 18 horas aproximadamente, desarrolló cuadro brusco de disnea, astenia, mal estado general, sudoración y dolor en hemitórax derecho, siendo ingresada en Urgencias y posteriormente en la Unidad de Cuidados Intensivos. Se realizó un electrocardiograma que mostraba ritmo sinusal y patrón S1, Q3. La analítica de sangre: $\mathrm{pH}=7,41, \mathrm{pCO}_{2}=36$ y $\mathrm{po}_{2}=60$. El ecocardiograma transtorácico mostraba ligera dilatación de cavidades derechas con signos de hipertensión pulmonar. El TAC helicoidal torácico con contraste demostraba la presencia del trombo intraluminal en la rama inferior de la arteria pulmonar derecha (Fig. 1). Se efectuó eco-doppler venoso de extremidades inferiores, no detectándose trombosis venosa y, sin otras anomalías. Con el diagnóstico de TEP agudo, se instauró tratamiento con perfusión continua de heparina no-fraccionada y posteriormente y dicumarol, mejorando clínicamente y siendo alta hospitalaria a los 15 días. Desde entonces asintomática y a tratamiento con dicumarol.

La fleboesclerosis de varices en las extremidades inferiores tiene un riesgo de mortalidad prácticamente nulo, la morbilidad o riesgo de complicación es baja. Las complicaciones mas habituales son poco frecuentes y leves y son: flebitis, varicoflebitis, pigmentación residual, necrosis superficial de la piel en el lugar de punción, lipotimía y março (1). Otras complicaciones de tipo grave y de muy rara presentación son: trombosis venosa profunda, embolia pulmonar, shock anafiláctico, parada cardiaca, inyección intraarterial, extravasación extensa del esclerosante con necrosis del tejido subcutáneo y otras (1).

En una revisión bibliográfica hemos encontrado muy pocas referencias bibliográficas de TEP en relación con fleboesclerosis de varices $(2,3)$. 


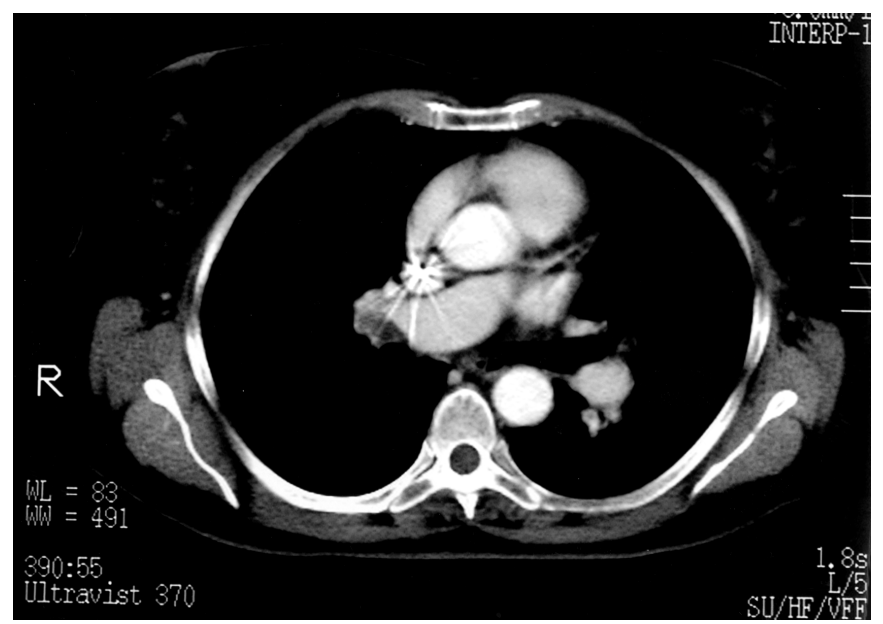

Fig. 1.

\section{Abad, M. Antúnez ${ }^{1}$, A. Cabrero²}

Servicio de Cirugía Cardiovascular-Angiología. Clínica Nuestra Señora del Perpetuo Socorro. ${ }^{\prime}$ Servicio de Medicina Interna y de ${ }^{2}$ Radiología. Clínica Santa Catalina. Las Palmas de Gran Canaria

1. Sánchez CF, Tropper U. Tratado de escleroterapia, flebectomía ambulatoria y ulceras venosas. Fundación Flebológica Argentina. Buenos Aires, 1996: 127-145.

2. Lee BB, Kim DI, Huh S, Kim HH, Choo IW, Byum HS. New experiences with absolute ethanol sclerotherapy in the management of a complex form of congenital venous malformation. J Vasc Surg 2001; 33: 764-772.

3. Harafi M, Orliaget G, Meyer P, Blanot S, Brunell F, Carli P. Embolie Pulmonaire au curs de la sclerotherapie percutaneé d'un angiome veineux sous anesthesie general chez un enfant. Annales Francaises d'Anesthesie et Reanimation 2001; 20: 556-558.

\section{Insuficiencia cardiaca: ¿sabemos realmente lo que estamos tratando?}

\section{Sr. Director:}

La insuficiencia cardiaca (IC) representa el estadío final común de la mayoría de las enfermedades cardiovasculares. El aumento progresivo de la esperanza de vida en las últimas décadas ha producido una elevación de la incidencia y prevalencia de esta patología. A pesar de los avances realizados en los últimos años en el tratamiento farmacológico de esta entidad, la variabilidad clínica y epidemiológica de este tipo de pacientes hace que los tratamientos pautados no sean homogéneos (debido en parte a los escasos estudios efectuados en pacientes con IC y función sistólica conservada). En nuestro centro (un hospital público de media y larga estancia con 150 camas de Medicina Interna, dependiente de la Consellería de Sanidad de la Comunidad Valenciana y ubicado en el área de salud 18 de la provincia de Alicante, en la comarca de l'Alacantí) hemos querido conocer el perfil y las características clínicas de los pacientes ingresados a cargo de Medicina Interna con el diagnóstico de IC. Para ello se realizó un registro desde octubre del 2002 hasta febrero del 2003 (cinco meses consecutivos) donde se recogieron datos sobre edad, sexo, clase funcional basal de la NYHA (previa al ingreso), enfermedades asociadas y etiología de la IC. Se practicó a todos los pacientes un ecocardiograma transtorácico (ETT) para valorar la fracción de eyección del ventrículo izquierdo (FEVI). Fueron incluidos en el estudio 50 pacientes, varones el $46 \%$ y mujeres el 54\% restante. La edad media fue de 76,3 años (entre 49 y 94 años), siendo similar para ambos sexos. El grado funcional basal de la NYHA hallado fue: clase I (6\%), clase II (46\%), clase III (44\%) y clase IV (4\%). En cuanto a las enfermedades asociadas más frecuentemente, predominaron: hipertensión arterial (70 \%), diabetes mellitus (42\%), dislipemia (32\%), EPOC (18\%), tabaquismo (10\%) y etilismo (4\%). Dentro de las etiologías responsables de la IC, la cardiopatía hipertensiva destaca con el $44 \%$ de los casos, seguida de la isquémica $(30 \%)$, valvular $(20 \%)$, miocardiopatía dilatada idiopática (4\%) y restrictiva (2\%). En el ETT se apreció una FEVI mayor del $45 \%$ (IC con función sistólica conservada) en el $72 \%$ de los casos, y una FEVI menor o igual al 45\% (IC con función sistólica deprimida) únicamente en el $28 \%$ restante. Tanto los datos de la edad como del sexo de nuestros pacientes difieren notablemente de los que son habituales en los ensayos clínicos (varones menores de 65 años) (1,2). Las etiologías de la IC y la clase funcional basal de la NYHA son similares a las halladas en el reciente registro del Grupo de Trabajo de IC de la Sociedad Española de Medicina Interna (SEMI) (1). Respecto a la FEVI, hemos encontrado que está conservada en el $72 \%$ de nuestros pacientes, pudiendo estar este llamativo dato relacionado con la gran comorbilidad asociada (70\% de HTA y $42 \%$ de DM), la edad avanzada y la exclusión habitual de los ancianos de la realización de ETT en los ensayos clínicos 2-4 (no en nuestro caso), provocando ésto un sesgo importante "a la baja" de los pacientes encuadrados en el grupo de IC diastólica o con función sistólica conservada. En los escasos estudios realizados en este tipo de pacientes, su diagnóstico se ha asociado básicamente al sexo femenino, la senilidad y la HTA (factores con alta incidencia en nuestro estudio) (2-5). A pesar de que actualmente el tratamiento de la IC con función sistólica conservada se ha extrapolado desde los estudios con pacientes que presentan una depresión de la función sistólica (5-7), debemos ser conscientes de qué tipo de pacientes estamos tratando habitualmente en los Servicios de Medicina Interna. Posiblemente en las Secciones de Cardiología se traten más pacientes jóvenes y con cardiopatía isquémica, y sus características se aproximen en mayor medida a los cánones habituales de los ensayos clínicos (3). Sin embargo, la senilidad y la pluripatología de los pacientes que ingresan con cargo a Medicina Interna, condicionan notablemente el tipo de asistencia y el tratamiento que se administra a unos enfermos que desafortunadamente no suelen aparecer reflejados en los ensayos clínicos.

\section{F. Camarasa García, M. I. Serrano Mateo, L. de Teresa Parreño}

Servicio de Medicina Interna. Hospital San Vicente. San Vicente del Raspeig (Alicante)

1. Grupo de Trabajo de Insuficiencia Cardiaca de la Sociedad Española de Medicina Interna (SEMI). La insuficiencia cardiaca en los servicios de medicina interna (estudio SEMI-IC). Med Clin (Barc) 2002; 118: 605-610.

2. Verdejo Bravo C. Manejo de la insuficiencia cardiaca del viejo. ¿Quién y dónde? Visión del geriatra . Rev Esp Geriatr Gerontol 2002; 37: 5-12.

3. Martínez-Sellés M, García Robles JA, Prieto L, et al. Características de los pacientes ingresados por insuficiencia cardiaca según el estado de su función ventricular. Rev Esp Cardiol 2002; 55: 579-586.

4. Permanyer Miralda G, Soriano N, Brotons C, et al. Características basales y determinantes de la evolución en pacientes ingresados por insuficiencia cardiaca en un hospital general. Rev Esp Cardiol 2002; 55: 571-578.

5. García MJ. Diagnóstico y guía terapéutica de la insuficiencia cardiaca diastólica. Rev Esp Cardiol 2003; 56: 396-406.

6. The Task Force of the Working Group on Heart Failure of the European Society of Cardiology. Guidelines for the diagnosis and treatment of heart failure . Eur Heart J 2001; 22: 1527-1560.

7. Agustí Escasany A, Durán Dalmau M, Arnau de Bolós JM, et al. Tratamiento médico de la insuficiencia cardiaca basado en la evidencia. Rev Esp Cardiol 2001; 54: 715-734. 